\title{
Common effects of emotional valence, arousal and attention on neural activation during visual processing of pictures
}

\author{
Richard D. Lane ${ }^{\mathrm{a}, \mathrm{b}, *}$, Phyllis M-L. Chua ${ }^{\mathrm{a}, \mathrm{c}}$, Raymond J. Dolan ${ }^{\mathrm{a}, \mathrm{d}}$ \\ ${ }^{\text {a } W e l l c o m e ~ D e p a r t m e n t ~ o f ~ C o g n i t i v e ~ N e u r o l o g y, ~ I n s t i t u t e ~ o f ~ N e u r o l o g y, ~ Q u e e n ~ S q u a r e, ~ L o n d o n ~ W C 1 N ~} 3 B G, U K$ \\ ${ }^{\mathrm{b}}$ Department of Psychiatry, University of Arizona College of Medicine, Tucson, AZ, USA \\ ${ }^{\mathrm{c}}$ Department of Psychiatry, The Royal Melbourne Hospital, Melbourne, Australia \\ ${ }^{\mathrm{d}}$ Academic Department of Psychiatry, Royal Free Hospital School of Medicine, London NW3, UK
}

Received 11 May 1998; accepted 4 January 1999

\begin{abstract}
Emotion and attention heighten sensitivity to visual cues. How neural activation patterns associated with emotion change as a function of the availability of attentional resources is unknown. We used positron emission tomography (PET) and ${ }^{15} \mathrm{O}$-water to measure brain activity in male volunteers while they viewed emotional picture sets that could be classified according to valence or arousal. Subjects simultaneously performed a distraction task that manipulated the availability of attentional resources. Twelve scan conditions were generated in a $3 \times 2 \times 2$ factorial design involving three levels of valence (pleasant, unpleasant and neutral), two levels of arousal and two levels of attention (low and high distraction).

Extrastriate visual cortical and anterior temporal areas were independently activated by emotional valence, arousal and attention. Common areas of activation derived from a conjunction analysis of these separate activations revealed extensive areas of activation in extrastriate visual cortex with a focus in right BA18 $(12,-88,-2)(Z=5.73, P<0.001$ corrected $)$ and right anterior temporal cortex BA38 $(42,14,-30)(Z=4.03, P<0.05$ corrected). These findings support an hypothesis that emotion and attention modulate both early and late stages of visual processing. (C) 1999 Elsevier Science Ltd. All rights reserved.
\end{abstract}

Keywords: Functional imaging; Extrastriate visual cortex; Anterior temporal cortex; Amygdala; Attention; Arousal

\section{Introduction}

Emotional arousal modifies the allocation of attentional resources and heightens sensitivity to environmental cues related to the motivational state induced by the provoking stimulus [30]. This is particularly true for stimuli with inherent significance for survival, a phenomenon referred to as 'natural selective attention' [22]. Conversely, the way in which attention is allocated during emotional arousal can significantly alter an emotional state $[31,36]$.

Although there is a growing literature on the func-

\footnotetext{
* Corresponding author. Department of Psychiatry, PO Box 245002, Tucson, AZ 85724-5002, USA; fax: + 1-520-626-2004.

E-mail address: lane@u.arizona.edu (R.D. Lane)
}

tional neuroanatomy of visual attention $[6,7,9,16]$, relatively little functional neuroimaging work has addressed how attention and emotion interact during visual processing. We recently demonstrated [21] that a manipulation of attention profoundly influenced neural activation while subjects processed emotional pictures. In that paradigm subjects attended either to the spatial or emotional aspects of each picture.

An alternative approach is to vary, through an independent manipulation, the attentional resources available during picture viewing. Dual task paradigms are a powerful means for varying allocation of attentional resources and have been used in a variety of functional neuroimaging studies [32,37]. By having subjects view pictures under conditions of high vs. low distraction, we examined how this manipulation of attention influ- 
enced neural activation patterns associated with processing emotional pictures.

One theory of emotion is that it is reducible to two fundamental dimensions, valence and arousal [23]. Valence refers to the direction of behavioral activation associated with emotion, either toward (appetitive motivation, pleasant emotion) or away from (aversive motivation, unpleasant emotion) a stimulus. Arousal is proposed to be orthogonal to valence and refers to the intensity of the emotional activation, ranging from excited to calm. To date, the neural correlates of emotional valence have been studied in a variety of functional neuroimaging paradigms $[12,19,20,27]$ but those of emotional arousal have not. Taking both dimensions of emotion into account is arguably necessary to determine whether attention interacts with emotion per se or with high arousal emotion in particular.

The right hemisphere has long been proposed to have a special role in both emotional [35] and attentional [33] processing. It has been suggested that the right hemispheric predominance in attention and emotion may be closely related [14,15], but the nature of this interaction and the exact location within the right hemisphere where such an interaction occurs has not been determined. By examining the neural correlates of emotional valence and arousal in the context of a dual task paradigm, we were able to examine the nature and location of the interaction between attention and the two proposed dimensions of emotion.

\section{Methods}

Subjects were six healthy right-handed males with no history of medical, neurological or psychiatric disorder with a mean age of 33.0 years $(\mathrm{SD}=7.8)$. None of the subjects were taking medication nor had a history of substance abuse. All had normal visual and auditory acuity.

Twelve scan conditions were generated from a $3 \times 2 \times 2$ factorial design. These factors included: (1) Valence: three levels; pleasant, unpleasant and neutral; (2) Arousal: two levels; high and low arousal; and (3) Attention: two levels; low and high distraction.

Emotional valence and arousal were determined by picture sets created using the International Affective Picture System [24]. Six types of picture sets were created by the three valence and two arousal conditions based on normative ratings in several hundred subjects. Each picture set consisted of 14 color pictures. Each picture was presented for $6 \mathrm{~s}$ followed by a blank screen for $1 \mathrm{~s}$ between pictures. Pleasant pictures included low arousal stimuli such as flowers or ice cream and high arousal stimuli such as female nudes or skydivers. Unpleasant pictures included low arousal stimuli such as a cemetery or garbage and high arousal stimuli such as a mangled face or a gun aimed at the viewer. Neutral stimuli included neutral faces, household objects and complex scenes such as a freeway. During the 30-s period of maximum signal acquisition the presence of faces and human figures was matched across neutral and emotional conditions. Pictures with letters or numbers were excluded. Picture conditions were counterbalanced across subjects to address potential order effects.

The distracter tasks consisted of matching keypad responses to auditory tones presented simultaneously during viewing of the picture sets. There were three keypad keys corresponding to three types of tones: low, medium and high pitched. A tone was presented every $1.25 \mathrm{~s}$. The low distraction condition involved tone presentation in a fixed sequence: low-mediumhigh-low-medium-high etc. The high distraction condition consisted of tone presentation in a random sequence. Subjects learned the distracter tasks prior to the scanning session and were told prior to each scan whether the tones would be presented in a fixed or random sequence. Before each scan they were instructed to attend to the pictures for later recognition testing while making accurate keypad responses. Accuracy and reaction time data from keypad responses were obtained on four subjects.

Electrodes were placed on the forearms for ECG measurement (lead I), the face for measurement of left corrugator and zygomatic EMG activity, and the hypothenar eminence of the left palm for skin conductance measurement. A skin conductance coupler provided a constant $0.5 \mathrm{~V}$ across electrodes. The data were sampled at $200 \mathrm{~Hz}$ and reduced off-line. Skin conductance responses (SCR) were scored as the largest response during the 6-s picture viewing period beginning 1-4 s after picture onset. A $\log$ transformation $[\log (\mathrm{SCR}+1)]$ was used to normalize the distribution of these responses. Skin conductance responses were not observed in one subject, therefore skin conductance data are presented on five subjects.

Following each scan subjects made a single rating of the pleasure and arousal of the entire picture sequence. The Self-Assessment Manikin (SAM) rating scales for pleasure and arousal [3] were used to make these ratings and were on display for the rating period only. Valence ratings ranged from 1 (very unhappy) to 9 (very happy) and arousal ratings ranged from 1 (very calm) to 9 (very excited). To evaluate whether subjects attended to the pictures during scanning, 21 pictures were presented immediately after the scan ended including the 14 pictures just presented, mixed with seven new pictures. Subjects indicated whether or not they had seen each picture during the scanning period.

Four of the subjects were also studied at a later date to evaluate any association between eye movements 
and scan conditions. The entire imaging protocol was duplicated in the scanner without radiotracer administration. EOG recordings of vertical and horizontal eye movements were obtained and counted manually offline.

Scans of the distribution of $\mathrm{H}_{2}{ }^{15} \mathrm{O}$ were obtained using a Siemens/CPS ECAT EXACT HR+ PET scanner operated in high sensitivity 3-D mode. Subjects received a total of $350 \mathrm{Mbq}$ of $\mathrm{H}_{2}{ }^{15} \mathrm{O}$ (about $8 \mathrm{mCi}$ ) over $20 \mathrm{~s}$ through a forearm cannula. Images were reconstructed into 63 planes, using a Hann filter, resulting in a $6.4 \mathrm{~mm}$ transaxial and $5.7 \mathrm{~mm}$ axial resolution (full width at half maximum, FWHM). The data were analyzed with statistical parametric mapping (SPM97) [10,11] using SPM software from the Wellcome Department of Cognitive Neurology, London, implemented in Matlab (Mathworks, Sherborn, MA). After initial realignment, the scans were transformed into a stereotactic space that uses a standard coordinate system [39]. The scans were smoothed using a Gaussian filter set at $16 \mathrm{~mm}$ FWHM. The regional cerebral blood flow (rCBF) equivalent measurements were adjusted to a global mean of $50 \mathrm{ml} / \mathrm{dl} / \mathrm{min}$. A blocked (by subject) ANCOVA model was fitted to the data at each voxel, with separate condition effects for each level of valence, arousal and attention. Predetermined contrasts of the condition effects at each voxel were assessed using the $t$ statistic (converted to $Z$ scores), giving a statistic image for each contrast. MRI scans of the head of each subject were co-registered with PET data for purposes of anatomical localization and transformed into this stereotactic space. Local maxima are reported in this stereotactic space. Findings that exceed a height threshold of $Z=3.09, P<0.001$ uncorrected are tabulated.

\section{Results}

\subsection{Behavioral data}

Valence ratings for pleasant $($ mean $=7.04 ; \mathrm{SD}=1.27)$ picture sets were significantly higher $(t=8.50 ; \mathrm{df}=5$; $P<0.001)$ and valence ratings of unpleasant $($ mean $=2.25 ; \mathrm{SD}=1.11)$ picture sets were significantly lower $(t=6.45 ; \mathrm{df}=5 ; P<0.001)$ than valence ratings of neutral picture sets $($ mean $=4.67 ; \mathrm{SD}=1.09)$. Selfreported arousal was also greater during pleasant $($ mean $=5.54 ; \mathrm{SD}=2.62 ; t=3.82 ; \mathrm{df}=5 ; P<0.01)$ and unpleasant $($ mean $=6.83 ; \mathrm{SD}=1.79 ; t=11.65 ; \mathrm{df}=5$; $P<0.001)$ than neutral picture sets $($ mean $=3.04$; $\mathrm{SD}=1.85$ ). Pleasant and unpleasant picture sets did not differ in arousal level $(t=1.88 ; \mathrm{df}=5$; NS). High arousal pleasant pictures (mean $=7.67 ; \mathrm{SD}=0.78)$ were more arousing $(t=11.12 ; \mathrm{df}=5 ; P<0.001)$ than low arousal pleasant pictures $($ mean $=3.42 ; \quad \mathrm{SD}=1.98)$. High arousal unpleasant pictures (mean $=7.67$; $\mathrm{SD}=1.37) \quad$ were more arousing $(t=5.42 ; \mathrm{df}=5$; $P<0.003$ ) than low arousal unpleasant pictures (mean $=6.00 ; \mathrm{SD}=1.81)$.

The valence and arousal values for the six types of picture sets from this sample were then compared to the valence and arousal values for males in the US (calculated from the US population norms for the individual pictures in each set) [24]. These normative values constituted the principal basis for selecting pictures for each picture set. Of the six comparisons, the only significant difference $(Z=2.10, \quad P<0.018)$ was that subjects in this sample rated the low arousal unpleasant picture sets as more arousing than US males. The correlations between the selfreported ratings of this sample and American norms (valence: $r=0.98, n=12, P<0.001$; arousal: $r=0.90$, $n=12, \quad P<0.001)$ were quite high. These findings confirm that the IAPS picture sets, which were created based on American norms, evoked comparable subjective responses in the British subjects we tested.

Skin conductance responses did not differ between the pleasant, unpleasant or neutral conditions, or between the low and high distraction conditions. However, skin conductance responses were greater during high arousal pleasant than low arousal pleasant conditions $(t=3.41, \mathrm{df}=4, P<0.02)$ and during high arousal emotional (pleasant and unpleasant) compared to low arousal conditions $(t=2.26, \mathrm{df}=4, P<0.05,1$ tailed).

The low distraction tasks (mean $=89.1 \%$ correct; $\mathrm{SD}=2.64$ ) were performed more accurately (keypad responses correctly matched to tones) $(t=4.48 ; \mathrm{df}=5$; $P<0.007)$ than were the high distraction $($ mean $=83.3 \%$ correct; $\mathrm{SD}=3.60)$ tasks. Similarly, reaction time was significantly greater $(t=7.59, \mathrm{df}=5$, $P<0.001)$ during the high $($ mean $=574.8 \mathrm{~ms}$; $\mathrm{SD}=26.0)$ compared to the low (mean $=452.3 \mathrm{~ms}$; $\mathrm{SD}=24.3 .3$ ) distraction tasks.

There were no differences in vertical or horizontal eye movements as a function of the valence or arousal level of the picture sets. However, there were more total eye movements during the low compared to the high distraction conditions $\left(F_{1,10}=8.98, P<0.01\right)$, attributable to more vertical movements $\left(F_{1,10}=10.5\right.$, $P<0.01)$.

To evaluate the effect of the distraction conditions on self-reported experience, we then compared the valence and arousal ratings of the six types of picture sets in the low and high distraction conditions. There were no significant differences.

Post-scan picture recognition accuracy percentage was very high (mean $=94.0 \%$, range $89.6-98.8 \%$, $\mathrm{SD}=6.66$ ) with no significant differences as a function 
Table 1

Regional cerebral blood flow (rCBF) increases during pleasant emotion (a), unpleasant emotion (b), valenced emotion (pleasant and unpleasant) (c), high arousal relative to low arousal conditions (including neutral stimuli) (d), high arousal relative to low arousal conditions (excluding neutral stimuli) (e), and low distraction relative to high distraction conditions (f). Local maxima are identified by name of structure, Brodmann's area and stereotactic coordinates. $x=$ distance $($ in $\mathrm{mm})$ to the right $(\mathrm{R})(+)$ or left $(\mathrm{L})(-)$ of midline; $y=$ distance anterior $(+)$ or posterior $(-)$ to the anterior commissure; $z=$ distance superior $(+)$ or inferior $(-)$ to a horizontal plane through the anterior and posterior commissures. $Z$ scores are normalized $t$-statistics that reflect the significance of the activation effect using SPM. All rCBF increases that exceed a threshold of $P<0.001$ uncorrected are displayed. The last column depicts the number of voxels in the cluster within which this local maximum is located. Superscripts indicate statistical significance of the height ( $Z$ score) or extent (cluster size) of the activation after correction for multiple comparisons $^{\mathrm{a}}$

\begin{tabular}{llllllll}
\hline Structure & Brodmann's area & $x$ & $y$ & $z$ & $Z$ score & Cluster size
\end{tabular}

(a) Pleasant

Putamen

Prefrontal cortex

$\mathrm{R}$ anterior temporal

L extrastriate

(b) Unpleasant

$\mathrm{R}$ extrastriate

(c) Valenced emotion (pleasant and unpleasant)

$\mathrm{L}$ extrastriate

$\mathrm{R}$ anterior temporal

$\mathrm{R}$ extrastriate

(d) High arousal (including neutral stimuli)

$\mathrm{R}$ extrastriate

L extrastriate

(e) High arousal (excluding neutral stimuli)

$\mathrm{R}$ extrastriate

$\mathrm{R}$ anterior temporal

Thalamus

Amygdala

Prefrontal

(f) Low distraction

Parahippocampal g.

Prefrontal cortex

$\mathrm{L}$ anterior temporal

Inferior temporal

Inferior frontal

Inferior temporal

Lingual g.

Parahippocampal g.

-30
-8
32
-8

18

$8-86$

$-12$

32

42

-90
10
-74

16

$-26$

$4.75^{*}$

3.54

3.28

$36-92$

-92
-94

14

$4.75^{*}$

4.22

$4993^{* * *}$

18

$$
-36
$$

36
38
-4
-26
8

-92
24
-18
0
62

14
-38
18
-10
36

$5.10^{* *}$

4.11

3.66

3.34

3.21
$11,493^{* * *}$
455
106
401
97

$\begin{array}{rrrrr}32 & -26 & -16 & 4.77^{*} & 8411^{* * *} \\ 0 & 60 & 38 & 4.59 & 2689^{* * *} \\ -36 & 16 & -24 & 4.25 & 478 \\ -54 & -24 & -20 & 3.76 & 116 \\ -44 & 42 & 4 & 3.57 & 204 \\ 54 & -18 & -30 & 3.55 & 146 \\ -10 & -80 & -22 & 3.32 & 182 \\ -28 & -40 & -14 & 3.10 & 17\end{array}$

${ }^{\mathrm{a}} P<0.05 ;{ }^{* *} P<0.01 ;{ }^{* * *} P<0.001$.

of valence or arousal. The recognition accuracy for each subject significantly exceeded the $50 \%$ accuracy expected by chance $(P<0.001)$, indicating that subjects attended to the pictures during the scans. Recognition accuracy was significantly lower $(t=3.18$; $\mathrm{df}=5, \quad P<0.025)$ following the high distraction (mean $=91.8 \%, \mathrm{SD}=4.2)$ compared to the low distraction conditions $($ mean $=96.3 \%, \mathrm{SD}=1.8)$. There were a total of 15 false positive (3\% error rate) and 74 false negative $(7.3 \%$ error rate) errors across the 72 scans, indicating that errors more commonly involved not recognizing pictures to which subjects had been previously exposed.

\subsection{Functional imaging data}

Neural activation patterns associated with processing emotional pictures are displayed in Table 1 . Pleasant emotion relative to neutral (Table 1a) was associated with activation in the putamen, medial prefrontal cortex, right anterior temporal cortex and left extrastriate visual cortex. Unpleasant emotion relative to neutral (Table $1 \mathrm{~b}$ ) was associated with activation in the right extrastriate visual cortex. Valenced emotion (pleasant and unpleasant emotion relative to neutral) (Table 1c) was associated with activation of bilateral extrastriate visual cortex and the right anterior tem- 
saggital

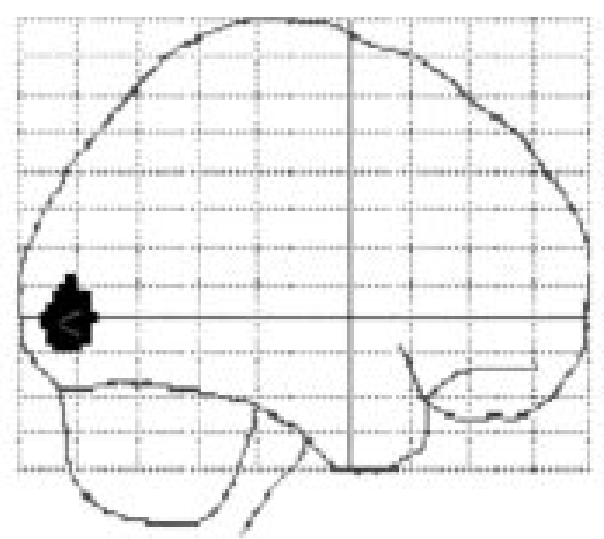

coronal

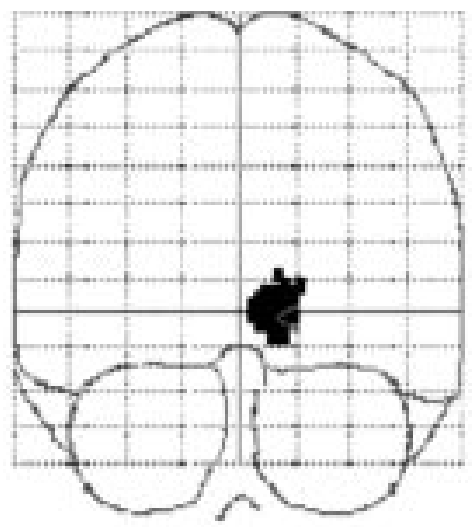

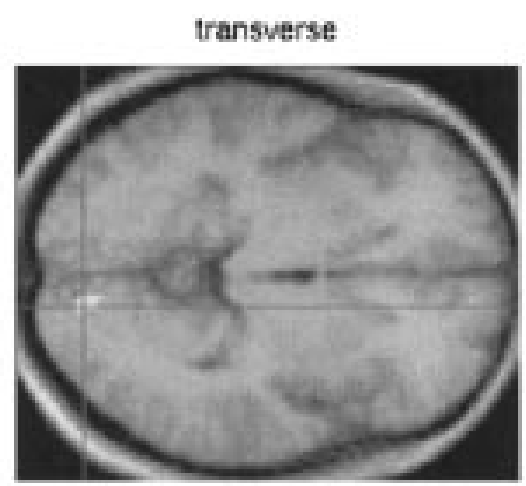

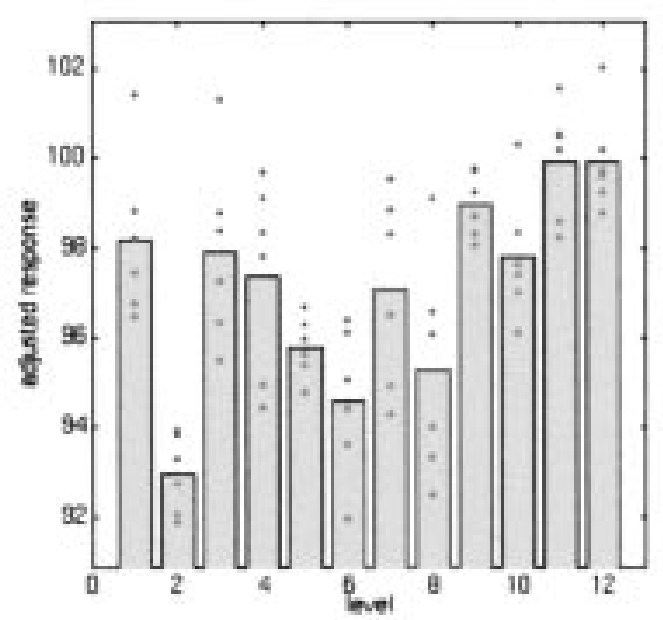

Fig. 1. A statistical parametric map (SPM) showing a statistically significant overlap in rCBF increases attributable to emotional valence (pleasant and unpleasant relative to neutral), arousal (high relative to low arousal) and attention (low relative to high distraction) in right extra-striate visual cortex (coordinates: $12,-88,-2)$. The figures in the upper right and upper left are projection images in the coronal and saggital planes, respectively. The transverse view in the lower left depicts the spatial distribution of the overlap in activation $(Z=5.73, P<0.001$, corrected) superimposed on the average structural MRI of the six male subjects. The figure in the lower right demonstrates blood flow values in each condition (pleasant: conditions 1-4; neutral: conditions 5-8; unpleasant: conditions 9-12; low distraction: odd numbered conditions; high distraction: even numbered conditions; low arousal: conditions 1, 2, 5, 6, 9, 10; high arousal: conditions 3, 4, 7, 8, 11, 12).

poral region; within the cluster which was maximal in left extrastriate cortex (BA18) a secondary activation was observed in right extrastriate visual cortex (BA18) (coordinates $=8,-86,4 ; Z=4.32, P<0.001$ uncorrected).

Processing high arousal relative to low arousal stimuli (including neutral stimuli) was associated with bilateral extrastriate visual cortical activation (Table $1 \mathrm{~d}$ ). Right anterior temporal activation was also observed (coordinates $=38,28,-34 ; Z=2.55, P<0.005$ uncorrected).

Processing high arousal pleasant and unpleasant stimuli compared to low arousal pleasant and unpleasant stimuli (excluding neutral stimuli) (Table 1e) was also associated with activation $(P<0.001$, corrected $)$ in right extrastriate visual cortex, as well as activations in right anterior temporal cortex, left amygdala, thalamus and medial prefrontal cortex (BA9).

Neural activation patterns associated with the distraction tasks are displayed in Table 1f. Across all emotion and neutral conditions, low distraction (relative to high distraction) was associated with activation of right parahippocampal gyrus and medial prefrontal cortex, left anterior temporal cortex, bilateral inferior temporal cortices, inferior frontal cortex, left lingual gyrus and left parahippocampal gyrus. Activations also included right anterior temporal cortex (coordinates $=40,16,-28, Z=3.20, P<0.001$ uncorrected) 
saggital

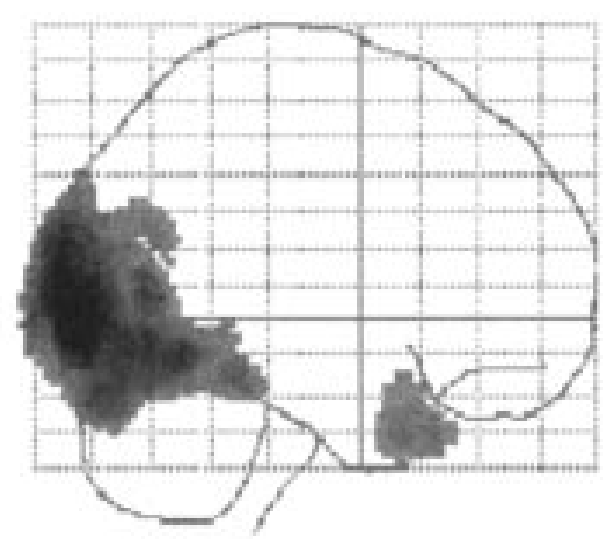

transverse

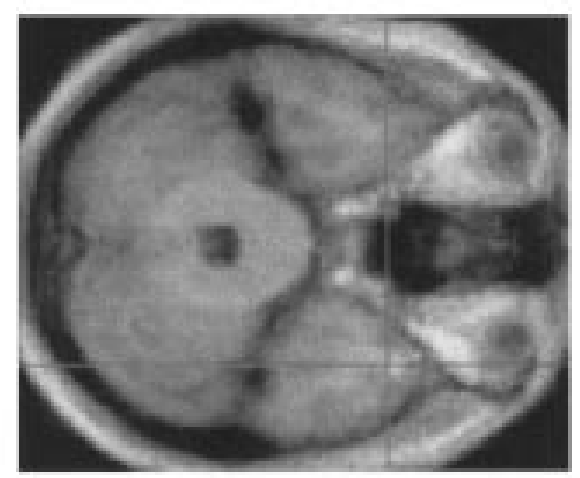

coronal
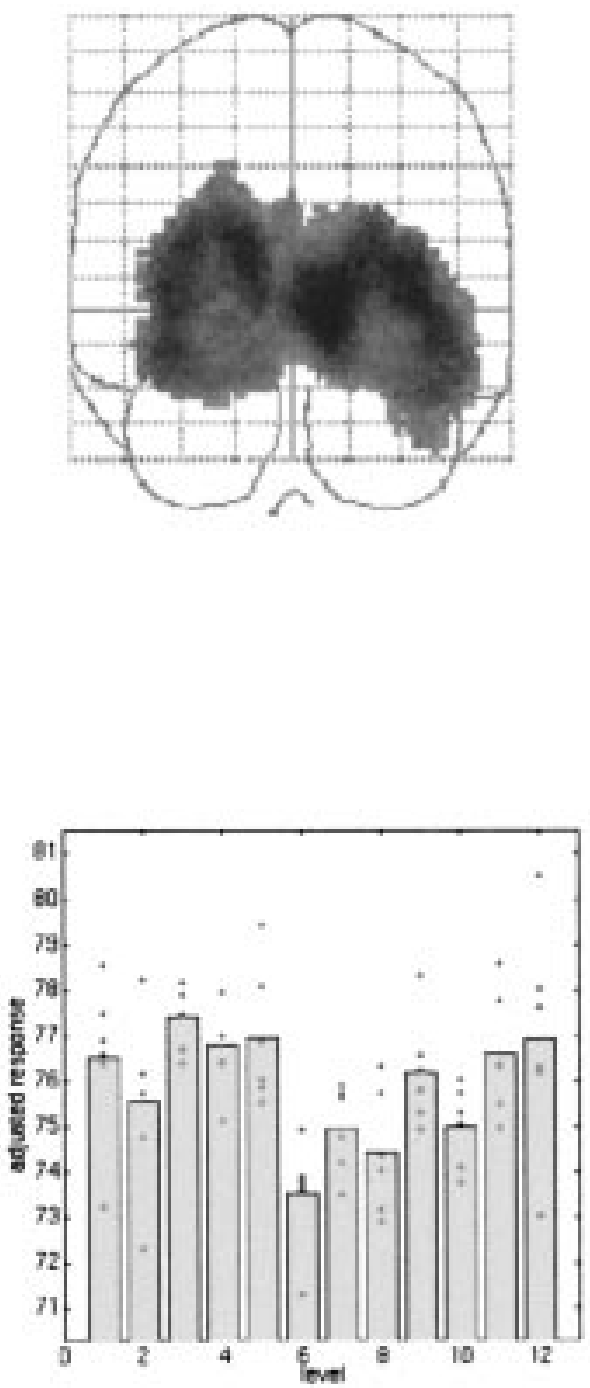

Fig. 2. A statistical parametric map (SPM) showing a statistically significant overlap in rCBF increases attributable to emotional valence (pleasant and unpleasant relative to neutral), arousal (high relative to low arousal) and attention (low relative to high distraction) in the right anterior temporal region. The figures in the upper right and upper left are projection images in the coronal and saggital planes, respectively. The transverse view in the lower left depicts the point of maximum overlap in the activations (coordinates: $42,14,-30 ; Z=4.03, P<0.001$, uncorrected) superimposed on the average structural MRI of the six male subjects. The cluster size ( 238 voxels, $P<0.05$ corrected) is depicted most clearly in the saggital view. Also evident is the overlap in activations in bilateral extrastriate visual cortex that exceeds a threshold of $P<0.001$ uncorrected. The figure in the lower right demonstrates blood flow values in each condition at this specific locus in the right anterior temporal region (pleasant: conditions 1-4; neutral: conditions 5-8; unpleasant: conditions 9-12; low distraction: odd numbered conditions; high distraction: even numbered conditions; low arousal: conditions 1, 2, 5, 6, 9, 10; high arousal: conditions 3, 4, 7, 8, 11, 12).

and right extrastriate visual cortex (coordinates $=40$, 92, $0 ; Z=4.27, P<0.001$, uncorrected).

A conjunction analysis was performed to determine where there was an overlap in activation patterns associated with emotional valence (Table 1c), arousal (Table $1 \mathrm{~d}$ ) and attention (Table 1f). This analysis revealed an extensive area of activation in extrastriate visual cortex with a focus in right BA18 $(12,-88,-2)$ $(Z=5.73, \quad P<0.001 \quad$ corrected; cluster size $=11,236$, $P<0.001$ corrected) (Fig. 1) and right anterior tem- poral region BA38 $(42,14,-30)(Z=4.03, P<0.001$ uncorrected; cluster size $=238, \quad P<0.05$ corrected) (Fig. 2).

Interaction analyses were performed to evaluate whether differences in activation between valenced emotion and neutral and between high and low arousal emotion varied as a function of the availability of attentional resources. There were no significant findings in predicted regions associated with the 2-way low distraction $\times$ emotional valence interaction, the 2-way 
low distraction $\times$ high arousal (excluding neutrals) interaction or the 3-way low distraction $\times$ high arousal (excluding neutrals) $\times$ emotional valence interaction.

\section{Discussion}

The purpose of this study was to evaluate how alterations in attentional resources influenced the activation patterns associated with the processing of emotion-evoking pictures. The finding of a common activation pattern associated with valence, arousal and attention in right extrastriate visual cortex and right anterior temporal cortex indicates common modulations of visual processing both at very early and late stages of cortical analysis.

Activation of extrastriate visual cortex has been demonstrated in several functional neuroimaging studies of emotion induced by visual stimuli $[4,20,25,28,34,40]$. Our observation of amygdala activation during emotional arousal is consistent with other findings [18]. Amaral [1] has shown that the amygdala sends reentrant projections to all levels of the visual-processing stream to an extent that exceeds afferent inputs to it. Morris and colleagues [28] have demonstrated that activation of the amygdala results in context dependent modulatory effects in extrastriate visual cortex. Specifically, the amygdala modulates extra-striate regions as a function of whether the current input consists of happy or fearful faces. The present study provides further evidence of a contextdependent modulatory effect on extrastriate cortex with respect to emotional valence, arousal and attention.

The current paradigm was specifically designed to examine the interaction between emotion and attention. A relative disadvantage of this approach is that emotional and neutral responses were always elicited in the context of a distracter task. It is likely that the resources available for processing the emotional stimuli were reduced by simultaneous performance of another task. Furthermore, the distracter tasks likely introduced a behavioral activation component in all conditions, including neutral. It may be speculated, then, that significant activation of the amygdala and thalamus emerged only in the high arousal conditions because higher intensity responses were needed to show a clear effect of emotion in the context of simultaneous distraction.

Single unit recordings in non-human primates [26] and a variety of functional neuroimaging studies in healthy volunteers have suggested that attention modulates activity of extra-striate visual cortex [38]. Attention has the effect of limiting the number of stimuli processed in a complex array, thus enhancing the perceptual processing of selected stimuli. This is anala- gous to the effect of emotional arousal on perceptual processing. Thus, the overlap in neural activation patterns between emotion and attention demonstrated in this study may correspond to their functional overlap at the behavioral level.

Activation of extrastriate visual cortex can occur as a result of increased visual stimulation introduced by eye movements [38]. However, we note that a recent fMRI study by Lang and colleagues [25] showed that extrastriate activity was greater during viewing of pleasant or unpleasant compared to neutral pictures, and that these effects were not attributable to eye movements. Our eye movement and functional neuroimaging data are consistent with these findings. The observation that the low distraction conditions were associated with more vertical eye movements relative to the high distraction conditions is consistent with other evidence that the distracter tasks constituted a successful manipulation of attention. Since the eye movement data were obtained after brain imaging was completed it is not possible to determine with certainty the extent to which vertical eye movements correlate with extrastriate activity.

A striking observation is that the right temporal pole is commonly activated by emotional valence, arousal and attention. This is a hierarchically higherorder visual cortical area that has reciprocal connections to amygdala, hippocampus (via perirhinal and entorhinal cortices) and prefrontal cortex [29]. This area has been activated during complex visual discrimination [29], retrieval of emotion-laden episodic memories [8] and attending to subjective emotional responses [21]. Electrical stimulation of this area in monkeys causes significant autonomic changes [17], consistent with its hypothesized role in emotional processes. The current findings indicate a role for anterior temporal cortex in processing the emotional meaning of visual stimuli. This evaluation might include retrieval of past emotional experiences for the purpose of evaluating the significance of the current stimulus and guiding the associated behavioral response. Attention may augment processing of this information at this late stage prior to its utilization in affect generation and response execution. It therefore appears that emotion and attention jointly contribute to the processing of visual information at a late stage of analysis, prior to the execution of a response to the environmental stimulus. The degree to which this occurs appears to be significantly compromised (or possibly inhibited) by distraction, as indicated by lower activity in the high distraction condition.

The right hemisphere has a predominant role, relative to the left, in the perception of emotional stimuli in the visual [2] and auditory [13] modalities and in the regulation of the autonomic activation associated with emotional arousal [41]. While the right hemisphere 
also preferentially participates in a network mediating attention [33], little is known about the extent to which these emotion-related and attention-related functions of the right hemisphere overlap. The rightsided convergence of emotional valence, arousal and attention effects seen in our study may reflect these right-sided asymmetries [14,15]. It is possible that during emotional arousal attentional mechanisms are automatically recruited, supporting the notion that the observed overlap in neural activation patterns exists because the same functional process is being studied in different contexts.

Medial prefrontal cortex was also activated in the low relative to high distraction conditions, in the high relative to low arousal conditions and in the pleasant relative to neutral conditions. This is an area that has been activated in previous PET studies of emotion $[19,20]$ and in a study of the neural correlates of intravenously-infused yohimbine [5], an alpha-2 antagonist that stimulates release of norepinephrine from the locus ceruleus. This area of medial prefrontal cortex is also very close to a region of the medial prefrontal cortex that we previously hypothesized participated in the representation of emotional experience [21]. The current findings indicate that activation in this area is associated with enhanced attention in the context of viewing emotional pictures, or, equivalently, deactivation during distraction. These findings together raise the possibility that the dampening of emotional experience with distraction is associated with a relative deactivation in this area. The failure to observe medial prefrontal activation in the unpleasant-minus-neutral comparison could be related to a possible aversive response to the distracter tasks, which could have rendered the neutral conditions somewhat unpleasant.

In conclusion, emotion is a mechanism that enables an organism to adapt psychologically, physiologically and behaviorally to meet environmental challenges. It has been well established through behavioral research that attention is altered during emotional arousal such that there is a heightened sensitivity to cues related to the current emotional state. In this study we have demonstrated that emotional valence, arousal and attention independently increase activity in extrastriate visual cortex and the anterior temporal region, suggesting that their common influence converges both early and late in visual analysis. These areas of convergence, and their lateralization to the right hemisphere, support an hypothesis that emotion and attention influence visual processing through shared mechanisms.

\section{Acknowledgements}

This work was supported by the Wellcome Trust amd MH00972 from NIMH to RDL.

\section{References}

[1] Amaral DG, Price JL, Pitkanen A, Carmichael ST. Anatomical organization of the primate amygdaloid complex. In: Aggleton J, editor. The Amygdala: Neurobiological Aspects of Emotion, Memory and Mental Dysfunction. New York: Wiley, 1992. p. $1-66$.

[2] Borod JC. Interhemispheric and intrahemispheric control of emotion: A focus on unilateral brain damage. Journal of Consulting and Clinical Psychology 1992;60:339-48.

[3] Bradley MM, Lang PJ. Measuring emotion: The SelfAssessment Manikin (SAM) and the semantic differential. Journal of Experimental Psychiatry \& Behavior Therapy 1994:25:49-59.

[4] Breiter HC, Etcoff NL, Whalen PJ, Kennedy WA, Rauch SL, Buckner RL, Strauss MM, Hyman SE, Rosen BR. Response and habituation of the human amygdala during visual processing of facial expression. Neuron 1996;17:875-87.

[5] Cameron OG, Zubieta JK, Grunhaus L, Minoshima S. Effects of yohimbine on cerebral blood flow, symptoms, and physiological functions in humans (submitted).

[6] Corbetta M, Miezin FM, Dobmeyer S, Shulman GL, Petersen SE. Attentional modulation of neural processing of shape, color, and velocity in humans. Science 1990;248:1556-9.

[7] Fink GR, Halligan PW, Marshall JC, Frith CD, Frackowiak RSJ, Dolan RJ. Where in the brain does visual attention select the forest and the trees? Nature 1996;382:626-8.

[8] Fink GR, Markowitsch HJ, Reinkemeier M, Bruckbauer T, Kessler J, Heiss W-D. Cerebral representation of one's own past: neural networks involved in autobiographical memory. Journal of Neuroscience 1996;16:4275-82.

[9] Frith C, Dolan RJ. Brain mechanisms associated with top-down processes in perception. Philosophical Transactions of the Royal Society of London Series B: Biological Sciences (London) 1997;352(1358):1221-30.

[10] Friston KJ, Ashburner J, Frith CD, Poline J-P, Heather JD, Frackowiak RS. Spatial registration and normalization of images. Human Brain Mapping Supplement 1995;3:165-89.

[11] Friston KJ, Holmes AP, Worsley KJ, Poline J-P, Frith CD, Frackowiak RS. Statistical parametric maps in functional imaging: a general linear approach. Human Brain Mapping Supplement 1995;2:189-210.

[12] George MS, Ketter TA, Parekh PI, Horowitz B, Herscovitch P, Post RM. Brain activity during transient sadness and happiness in healthy women. American Journal of Psychiatry 1995;152:341-51.

[13] Heilman KM, Scholes R, Watson RT. Auditory affective agnosia-disturbed comprehension of affective speech. Journal of Neurology Neurosurgery and Psychiatry 1975;38:69-72.

[14] Heilman KM, Van Den Abell T. Right hemispheric dominance for mediating cerebral activation. Neuropsychologia 1979;17:315-21.

[15] Heilman KM. The neurobiology of emotional experience. Journal of Neuropsychiatry and Clinical Neurosciences 1997;9:439-48.

[16] Heinze HJ, Mangun GR, Burchert W, Hinrichs H, Scholz M, Münte TF, Gös A, Scherg M, Johannes S, Hundeshargen, Gazzaniga MS, Hillyard SA. Combined spatial and temporal imaging of brain activity during visual selective attention in humans. Nature 372:543-546.

[17] Kaada BR. Cingulate, posterior orbital, anterior insular and temporal pole cortex. In: Field J, Magoun HW, Hall VE, editors. Handbook of Physiology: Neurophysiology Vol II, Section 1. Washington DC: American Physiological Society, 1960. Chapter 55, p. 1345-1372.

[18] Kapp BS, Whalen PJ, Supple WF, Pascoe JP. Amygdaloid con- 
tributions to conditioned arousal and sensory information processing. In: Aggleton J, editor. The Amygdala: Neurobiological Aspects of Emotion, Memory and Mental Dysfunction. New York: Wiley, 1992. p. 229-54

[19] Lane RD, Reiman EM, Ahern GL, Schwartz GE, Davidson RJ. Neuroanatomical correlates of happiness, sadness, and disgust. American Journal of Psychiatry 1997;154:926-33.

[20] Lane RD, Reiman EM, Bradley MM, Lang PJ, Ahern GL, Davidson RJ, Schwartz GE. Neuroanatomical correlates of pleasant and unpleasant emotion. Neuropsychologia 1997;35:1437-44.

[21] Lane RD, Chua PM-L, Dolan R. Neural activation during selective attention to subjective emotional responses. Neuroreport 1997;8:3969-72.

[22] Lang PJ, Bradley MM, Cuthbert MM. Motivated attention: Affect, activation and action. In: Lang PJ, Simons RF, Balaban MT, editors. Attention and Orienting: Sensory and Motivational Processes. Hillsdale, New Jersey: Lawrence Erlbaum Associates, 1997.

[23] Lang PJ, Greenwald MK, Bradley MM, Hamm AO. Looking at pictures: Affective, facial, visceral and behavioral reactions. Psychophysiology 1993;30:261-73.

[24] Lang PJ, Bradley MM, Cuthbert BN. The International Affective Picture System (IAPS): Photographic Slides. The Center for Research in Psychophysiology, Gainesville, University of Florida, 1995.

[25] Lang PJ, Bradley MM, Fitzsimmons JR, Cuthbert BN, Scott JD, Moulder B, Nangia V. Emotional arousal and activation of the visual cortex: an fMRI analysis. Psychophysiology 1998;35:199-210.

[26] Luck SJ, Chelazzi L, Hillyard SA, Desimone R. Neural mechanisms of spatial selective attention in areas of V1, V2 and V4 of macaque visual cortex. Journal of Neurophysiology 1997;77:24-42.

[27] Morris JS, Frith CD, Perrett DI, Rowland D, Young AW, Calder AJ, Dolan RJ. A differential neural response in the human amygdala to fearful and happy facial expressions. Nature 1996;383:812-5.

[28] Morris JS, Friston KJ, Buchel C, Frith CD, Young AW, Calder AJ, Dolan RJ. A neuromodulatory role for the human amygdala in processing emotional facial expressions. Brain 1998;121:47-57.
[29] Nakamura K, Kubota K. The primate temporal pole: its putative role in object recognition and memory. Behavioural Brain Research 1996;77:53-77.

[30] Niedenthal PM, Kitayama S. The Heart's Eye-Emotional Influences in Perception and Attention. New York: Academic Press, 1994.

[31] Nix G, Watson C, Pyszczynski T, Greenberg J. Reducing depressive affect through external focus of attention. Journal of Social and Clinical Psychology 1995;14:36-52.

[32] Passingham RE. Attention to action. Philosophical Transactions of the Royal Society of London Series B: Biological Sciences (London) 1996;351(1346):1473-9.

[33] Posner MI, Dehaene S. Attentional networks. Trends in Neurosciences 1994;17:75-9.

[34] Reiman EM, Lane RD, Ahern GL, Schwartz GE, Davidson RJ, Friston KJ, Yun L-S, Chen K. Neuroanatomical correlates of externally and internally generated human emotion. American Journal of Psychiatry 1997;154:918-25.

[35] Ross ED. The aprosodias: Functional-anatomical organization of the affective components of language in the right hemisphere. Archives of Neurology 1981;38:561-9.

[36] Rothbart MK, Ziaie H, O'Boyle CG. Self-regulation and emotion in infancy. In: Eisenberg N, Fabes RA, editors. Emotion and its Regulation in Early Development. San Francisco: Jossey-Bass, 1992. p. 7-23.

[37] Shallice T, Fletcher P, Frith CD, Grasby P, Frackowiak RS, Dolan RJ. Brain regions associated with acquisition and retrieval of verbal episodic memory. Nature 1994;368:633-5.

[38] Shulman GL, Corbetta M, Buckner RL, Raichle ME, Fiez JA, Miezin FM, Petersen SE. Top-down modulation of early sensory cortex. Cerebral Cortex 1997;7:193-206.

[39] Talairach J, Tournoux P. Co-planar Stereotaxic Atlas of the Human Brain. New York: Thieme Medical Publishing, 1988.

[40] Wik G, Fredrikson M, Eriksson L, Stone-Elander S, Greitz T. A functional cerebral response to frightening visual stimulation. Psychiatry Research: Neuroimaging 1993;50:15-24.

[41] Wittling W. Brain asymmetry in the control of autonomic physiologic activity. In: Davidson RJ, Hugdahl K, editors. Brain Asymmetry. Cambridge, MA: MIT Press, 1995. p. 30557. 